\title{
Evaluation of silicon tuning fork resonators under mechanical loads and space-relevant radiation conditions
}

\author{
Tobias Bandi, ${ }^{a, b, c, \star}$ Jacek Baborowski, ${ }^{b}$ Alex Dommann, ${ }^{d}$ Herbert R. Shea, ${ }^{c}$ Francis Cardot, $^{\mathrm{b}}$ and Antonia Neels ${ }^{\mathrm{e}}$ \\ ${ }^{a} E P F L$, Swiss Space Center, 1015 Lausanne, Switzerland \\ ${ }^{b}$ CSEM, Microsystems Technology Division, Jaquet Droz 1, 2002 Neuchâtel, Switzerland \\ ${ }^{\circ} E P F L$, Microsystems for Space Technologies Laboratory, Rue de la Maladière 71b, 2002 Neuchâtel, Switzerland \\ ${ }^{d}$ Empa, Department Materials Meet Life, Lerchenfeldstrasse 5, 9014 Sankt Gallen, Switzerland \\ eEmpa, Center for X-ray Analytics, Überlandstrasse 129, 8600 Dübendorf, Switzerland
}

\begin{abstract}
This work reports on mechanical tests and irradiations made on silicon bulk-acoustic wave resonators. The resonators were based on a tuning fork geometry and actuated by a piezoelectric aluminum nitride layer. They had a resonance frequency of $150 \mathrm{kHz}$ and a quality factor of about 20,000 under vacuum. The susceptibility of the devices to radiation-induced degradation was investigated using ${ }^{60} \mathrm{Co} \gamma$-rays and $50 \mathrm{MeV}$ protons with space-relevant doses of up to $170 \mathrm{krad}$. The performance of the devices after irradiation indicated a high tolerance to both ionizing damage and displacement damage effects. In addition, the device characteristics were evaluated after mechanical shock and vibration tests and only small effects on the devices were observed. In all experiments, no significant changes of the resonance characteristics were observed within the experimental uncertainty, which was below $100 \mathrm{ppm}$ for the resonance frequency. The results support the efforts toward design and fabrication of highly reliable MEMS devices for space applications. $\odot 2014$ Society of Photo-Optical Instrumentation Engineers (SPIE) [DOI: 10.1117/1.JMM.13.4.043019]
\end{abstract}

Keywords: bulk-acoustic wave resonator; proton radiation; total dose test; radiation; micro-electromechanical systems; reliability. Paper 14010P received Jan. 29, 2014; accepted for publication Oct. 21, 2014; published online Dec. 18, 2014.

\section{Introduction}

Historically, the appearance of steels with constant elasticity over a wide temperature range, such as Elinvar, has made it possible to fabricate tuning fork resonators with high frequency stability in the ppm range. ${ }^{1,2}$ Currently, quartz resonators are the state-of-the-art but competition from siliconbased microfabricated devices is growing due to the possibility of highly parallel batch processing, low fabrication tolerances, excellent material quality, and high reliability. ${ }^{3}$ This is exemplarily illustrated in Table 1 , which shows a comparison of various commercially available real-time clock (RTC) oscillators. The last two entries are developments made by Swiss Center for Electronics and Microtechnology (CSEM) and partnering institutions which are close to commercialization. This table shows that the trend of RTC oscillators is going toward low single-digit frequency stability, combined with ultra-low power consumption levels of $1 \mu \mathrm{W}$ and below. In terms of power consumption and frequency, stability aluminum nitride (AIN) activated silicon resonators, based on the same technology as the resonators investigated in this work, are competitive in this segment.

The applicability of MEMS resonators in space depends on their ability to operate reliably under the demanding environmental conditions imposed by the spacecraft's trajectory. In this work, mechanical tests and irradiations were made on piezoelectrically activated silicon bulk-acoustic wave resonators in order to assess the devices' tolerance to these aspects of the hazardous environmental conditions in space. Radiationinduced degradation is one of the main differences between the environments on Earth and in space and can cause severe impairment of the device performance. The tolerance of MEMS to radiation depends on the operation principle, the design, and the material selection. In consequence of the multisided MEMS typology, a wide range of radiation effects and tolerances has been reported and design rules for enhanced radiation tolerance have been proposed. ${ }^{12}$ The majority of degradation effects are caused by ionizing damage, especially charge trapping in dielectrics, and structural defects induced by nonionizing energy losses (NIEL). Radiation effects have been extensively studied in quartz, where the motion of impurities and displacement damage may influence the elastic constants of the material. For a review on this topic, we refer to Ref. 13 and references therein. The influence of radiation on the mechanical properties of silicon has been studied by neutron irradiation, ${ }^{14}$ $\gamma$-ray and electron irradiation, ${ }^{15}$ and proton tests. ${ }^{16,17}$ However, to our knowledge, no studies on the influence of spacerelevant radiation on silicon-based resonators with frequency stabilities on the ppm-level have been presented to date.

This paper is structured as follows: first, relevant radiation conditions for space applications are summarized and the choice of radiation sources is discussed. Then the fabrication of the MEMS devices and the experimental setup for the dynamical characterization of the resonators are described. The following section contains the specifications of the radiation and mechanical tests, before the results are presented and discussed.

\section{Choice of Sources for Radiation Hardness Assessment}

The composition, flux density, and energy of the radiation are subject to considerable variations over time and orbit, 
Table 1 Comparison of real-time clock resonators with an output frequency of $32.768 \mathrm{kHz}$.

\begin{tabular}{|c|c|c|c|c|c|}
\hline Manufacturer/laboratory & Material & Frequency stability & Size $\left(\mathrm{mm}^{3}\right)$ & Power consumption & References \\
\hline Maxim Integrated & Not specified & $\pm 5 \mathrm{ppm}$ & $5.0 \times 4.0 \times 1.5$ & $2 \mu \mathrm{A}(2.3$ to $5.5 \mathrm{~V})$ & 4 \\
\hline SiTime & Silicon & $\pm 10 \mathrm{ppm}$ & $1.5 \times 0.8 \times 0.55$ & $0.75 \mu \mathrm{A}(1 \mathrm{~V})$ & 5 \\
\hline Fortiming Corp & Quartz & $\pm 3 \mathrm{ppm} /$ year & $1.5-\mathrm{mm}$ radius $9.4-\mathrm{mm}$ length & $1 \mu \mathrm{W}$ & 6 \\
\hline Silicon Labs & Silicon & \pm 10 ppm & $2 \times 2.5 \mathrm{~mm}^{2}$ footprint & $1.7 \mathrm{~mA}(1.7$ to $3.6 \mathrm{~V})$ & 7 \\
\hline Epson & Quartz & $\pm 5 \mathrm{ppm}$ & $3.6 \times 2.8 \times 1.2$ & $0.35 \mu \mathrm{A}(3 \mathrm{~V})$ & 8 \\
\hline Microcrystal & Quartz & \pm 20 ppm & $2.0 \times 1.2 \times 0.60 \mathrm{~mm}^{3}$ & $0.5 \mu \mathrm{W}$ (max.) & 9 \\
\hline $\begin{array}{l}\text { Project Go4Time within EC FP7 } \\
\text { research program (Quartz-resonator) }\end{array}$ & Quartz & \pm 2 ppm & $\begin{array}{c}1.5 \times 1.1 \times 0.7 \text { (targeted on } \\
\text { wafer-level) }\end{array}$ & $0.4 \mu \mathrm{A}(1.0$ to $3.3 \mathrm{~V})$ & 10 \\
\hline CSEM & $\begin{array}{l}\text { AIN activated } \\
\text { silicon resonator }\end{array}$ & $\pm 10 \mathrm{ppm}$ & $2.75 \times 0.75 \times 0.5$ (resonator) & $2 \mu \mathrm{A}(1 \mathrm{~V})$ & 11 \\
\hline
\end{tabular}

and radiation hardness assessment tests can only approximate the conditions in operation. ${ }^{18,19}$ The main contributions to radiation damage are made by electrons, protons, and electron-induced Bremsstrahlung. Due to the complexity and variability of conditions encountered during space missions, the effect of radiation is commonly simulated by a limited number of radiation sources and energies, reproducing the total doses absorbed over the device lifetime (e.g., conditions specified in Ref. 20). The total ionizing damage dose absorbed by a space system shielded by $4 \mathrm{~mm}$ of aluminum is on the order of $1 \mathrm{krad} /$ year in low earth orbits and $10 \mathrm{krad} /$ year in geostationary orbits. ${ }^{21}$ The total dose level usually requested in the qualification testing of generic electrical, electronic, and electromechanical (EEE) parts is $100 \mathrm{krad} .{ }^{20}$ For comparison, the expected ionizing dose of a tracking detector in the Large-Hadron-Collider of the European Organization for Nuclear Research (CERN) is 1 Mrad, obtained after 1.5 years of operation, which is much higher than most space doses. ${ }^{22}$

Figure 1 shows the linear energy transfer (LET) of different radiation types versus the particle energy. The LET

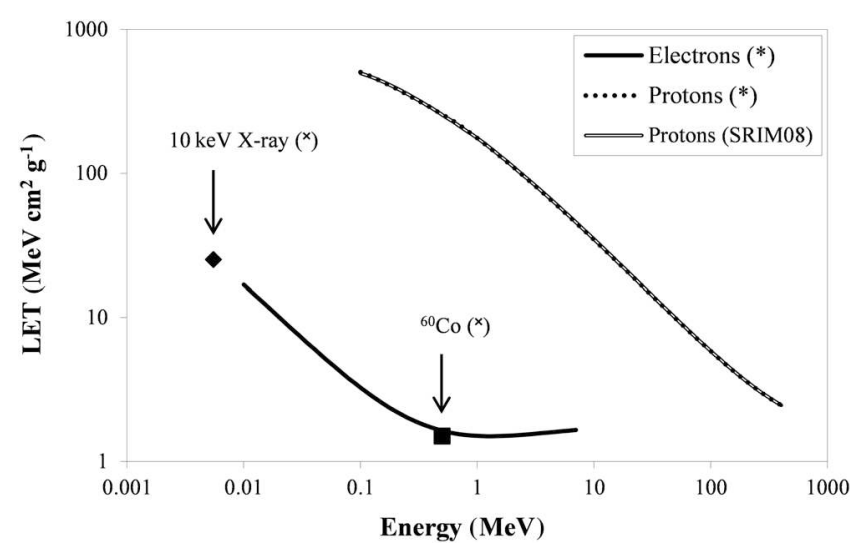

Fig. 1 Electronic stopping power of protons, electrons, and secondary electrons created by $\gamma$-rays and $10 \mathrm{keV} x$-rays. This figure is adopted from graphs in Refs. 23 and 24 and is containing data from Ref. $24(\times)$ and Ref. $25\left(^{*}\right)$, as well as SRIM-2008 calculations. denotes the ionizing energy deposited per unit length in the track of an impinging particle and is normalized by the material density. The energy range of the proton and electron sources corresponds to the energies observed in space. ${ }^{18}$

A commonly used source of ionizing damage is $\gamma$-rays produced by the radioactive decay of ${ }^{60} \mathrm{Co}$. The decay to ${ }^{60} \mathrm{Ni}$ occurs under the emission of photons with energies of 1.17 and $1.33 \mathrm{MeV}$. Gamma-radiation-induced damage is mainly caused by the Compton effect leading to direct ionization and energy deposition by the secondary electrons. The secondary electron energy is in the range of 0.1 to $1 \mathrm{MeV}^{26,27}$ In devices where the radiation-induced damage is correlated with ionization effects, the ${ }^{60} \mathrm{Co} \gamma$-rays are expected to produce similar effects as electron irradiation because the spectrum of $\gamma$-ray induced secondary electrons closely resembles space-relevant electron spectra. ${ }^{24}$ In analogy to this, $10 \mathrm{keV}$ x-rays are expected to reproduce ionization-mediated degradations caused by $\sim 10 \mathrm{MeV}$ protons. $^{28}$ This hypothesis has been studied in detail in metal-oxidesemiconductor (MOS) devices, which are mainly affected by charge trapping in thin oxide layers. In Refs. 29 and 30, the threshold voltage shift of MOS transistors has been evaluated under irradiation with protons, electrons, and ${ }^{60} \mathrm{Co} \gamma$-rays at doses of up to $500 \mathrm{krad}\left(\mathrm{SiO}_{2}\right)$. Although the radiation-induced change of the threshold voltage differed significantly between $41.4 \mathrm{MeV}$ protons and $\gamma$-rays a strong correlation between $10 \mathrm{keV}$ x-rays and protons was found. These observations have been attributed to variances in the charge yield and in the electron-hole pair distributions. $^{29}$ The fraction of electron-hole pairs that escape the initial recombination in the first fractions of a second depends on the electric field in the material and the distribution of electron-hole pairs. Impinging particles with high stopping powers create a dense population of ionization events, whereas for lower stopping powers the mean distance between electron-hole pairs is larger. Therefore, the charge yield is larger for radiation with lower stopping power than for radiation with higher stopping power and radiations creating similar distributions of ionization events affect irradiated specimen in a similar way. These experiments indicated 
that degradation effects due to ionization can be regarded as equivalent between ${ }^{60} \mathrm{Co} \gamma$-rays and electrons with space-relevant energies because the two radiation sources have similar LET. $^{29,30}$

NIEL cause the displacement of atoms and the creation of vacancies, interstitials, and extended damage zones. These structural defects may be electrically active, influencing the carrier mobility and minority carrier lifetime. ${ }^{12}$ Displacement damage is predominantly caused by heavy ions and protons, but also the secondary electrons generated by ${ }^{60} \mathrm{Co} \gamma$-rays carry enough energy to displace atoms from their lattice position and create recoils. The displacement damage induced by ${ }^{60} \mathrm{Co}$ radiation has been investigated theoretically on the basis of the energy distribution of secondary electrons created by Compton scattering of $\gamma$ rays. $^{26,27}$ However, the obtained NIEL differed significantly between the two studies. The displacement damage factor ratio $K_{\mathrm{e}} / K \gamma$ between $1 \mathrm{MeV}$ protons and ${ }^{60} \mathrm{Co}$ radiation was found to be 2.4 in Ref. 26 and 302 in Ref. 27, and the reason for this discrepancy was unclear to the authors of the latter paper. Nevertheless, the total displacement damage induced by electrons and $\gamma$-rays is orders of magnitude lower than proton-induced damages (Fig. 2).

Heavy ions are less abundant but are important sources of single-event effects due to their large stopping power. ${ }^{19}$ However, the feature sizes of the MEMS tuning fork resonators investigated here are on the micron-scale and the operation does not rely on thin depletion regions or $\mathrm{p}-\mathrm{n}$ junctions. Therefore, single-event effects are not expected to affect the devices.

Based on these considerations, the ${ }^{60} \mathrm{Co} \gamma$-rays and protons were selected for the radiation hardness assessment tests on the silicon tuning fork resonators.

\section{Resonator Design, Fabrication, and Characterization}

The tuning fork resonators were fabricated on silicon-oninsulator wafers. Their mode of operation and functional layout were based on resonators previously presented by CSEM. ${ }^{31-33}$ The fork tines were patterned into the device layer by deep reactive ion etching. They had a length of $900 \mu \mathrm{m}$, a thickness (in the plane of oscillation) of $100 \mu \mathrm{m}$, and a width of $22 \mu \mathrm{m}$ (Fig. 3). A one-micron thick layer of

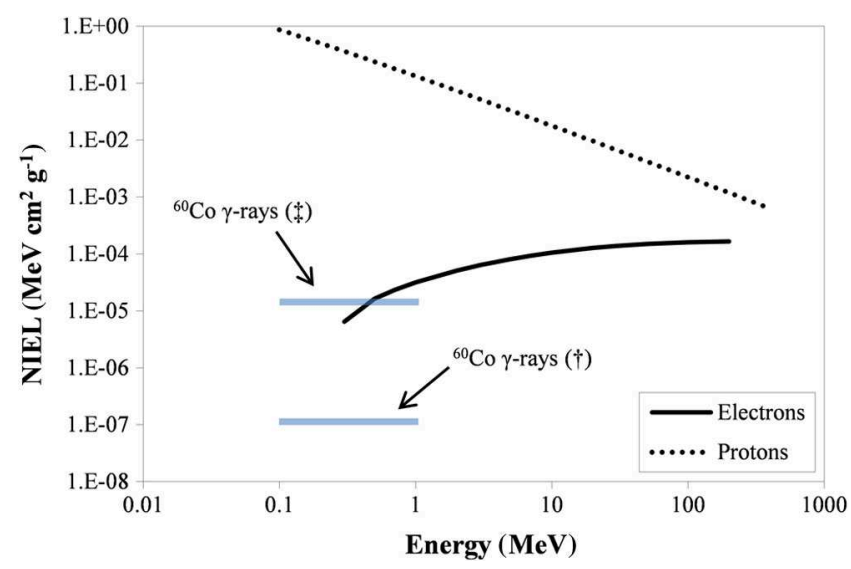

Fig. 2 Nonionizing energy losses for electron, proton and ${ }^{60} \mathrm{Co}$ radiation versus the particle energy. For ${ }^{60} \mathrm{Co}$, the energy of the secondary electrons is shown for Ref. $26(\ddagger)$ and Ref. $27(\dagger)$.
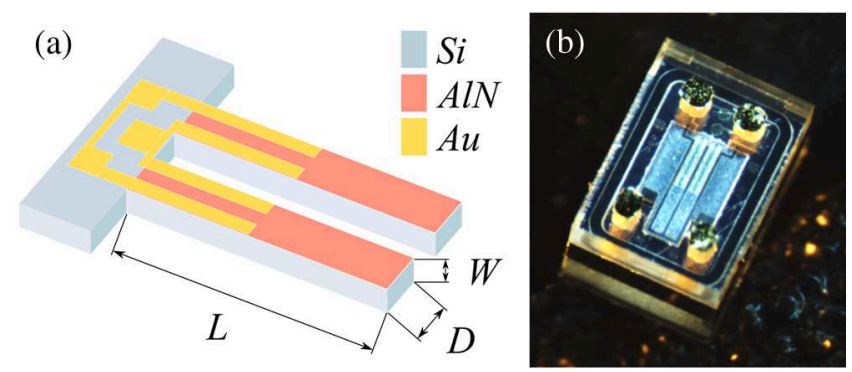

Fig. 3 (a) Schematic view of the tuning fork resonators (only the silicon-on-insulator device layer is shown). The length $L$ of the resonators was $900 \mu \mathrm{m}$, the width $W$ (normal to the direction of the oscillation) was $22 \mu \mathrm{m}$ and the thickness $D$ was $100 \mu \mathrm{m}$. (b) Optical image of an encapsulated resonator. The size of the package is $1.5 \times 2 \times 1.5 \mathrm{~mm}^{3}$.

piezoelectric AlN, with metallic top and bottom electrodes, was used to drive the resonators. The devices were waferlevel packaged by eutectic bonding using a glass cap wafer with through-glass-vias and the dies were then separated by diamond blade dicing. In the electrically active mode, the tines oscillated in the plane of the wafer.

The electrical characterization of the devices was made under controlled conditions using a measurement test setup as shown in Fig. 4. The impedance of the resonators was measured by sweeping the excitation frequency across the resonance and recording the impedance's absolute value and phase using a HP4192A impedance analyzer. A LabView ${ }^{\mathrm{TM}}$ program on a personal computer was used to control the impedance analyzer.

The response of the piezoelectric resonators to the driving (AC) voltage was modeled by the Butterworth-van-Dyke (BvD) equivalent circuit. ${ }^{32,34}$ Such a circuit is characterized by four parameters which account for the mass, the elasticity, and the damping of the mechanical resonator and for the electrical capacitance between the electrodes used for the actuation of the piezoelectric layer. From the impedance curves, the four BvD parameters were extracted by nonlinear fitting and were then used to calculate the resonance frequency and quality factor of the resonators.

The electromechanical properties of the resonators were evaluated under vacuum (pressure $<5 \times 10^{-3}$ mbar) where the resonance was not affected by air damping. The resonators had a resonance frequency of $150 \mathrm{kHz}$ and a quality factor of about 20,000. Throughout the measurements, the temperature and pressure of the chamber were monitored. The temperature dependency of the resonance frequency was $-3.5( \pm 0.5) \mathrm{Hz} / \mathrm{K}(-23 \mathrm{ppm} / \mathrm{K})$ while no temperature-dependent variation of the quality factor was observed. A correction was applied to the resonance frequencies normalizing to a reference temperature of $25^{\circ} \mathrm{C}$. Neither the resonance frequency nor the quality factor was affected by a DC bias $(0 \pm 5 \mathrm{~V})$. The measurement repeatability was composed from contributions from the error of the measurement technique, the fitting procedure, and the variations of the experimental conditions (e.g., humidity, temperature). For the purpose of evaluating the repeatability uncertainty of the experiments, the impedance characteristics of 34 devices were recorded three times with 1 week time between the measurements. The pooled standard deviation of the resonance frequency was $1.2 \mathrm{~Hz}$, corresponding to $11 \mathrm{ppm}$. The repeatability uncertainty of the quality factor was 450 . 


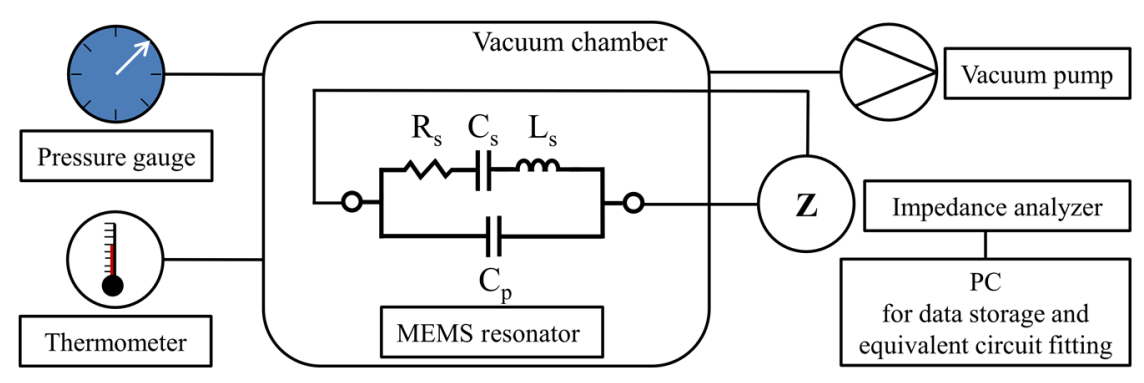

Fig. 4 Schematic view of the measurement test setup.

During the proton irradiation tests another impedance analyzer was used (Agilent 4292A). The repeatability uncertainty of this setup was inferior to the other setup due to a lower frequency resolution $(2.5 \mathrm{~Hz}$ instead of $0.2 \mathrm{~Hz})$. The repeatability uncertainty of the resonance frequency and the $Q$-factor was estimated to be $75 \mathrm{ppm}$ and 2500, respectively.

Figure 5 shows a measurement of the phase and magnitude of the impedance of a tuning fork resonator near the operation frequency. The fitted curve of the BvD equivalent circuit is shown in the same graph. Good fits were obtained near the antiresonance where the amplitude of the impedance had a minimum. At the resonance where the two tines moved in phase and the electrical impedance was at a maximum the fitted line deviated from the experiment. This was attributed to parasitic elements in the circuit and the fact that the impedance was close to the specified limit of the impedance analyzer. However, it is noted that the tuning fork resonators are commonly operated in the antiresonance mode where a good agreement between the fit and the measurements was obtained. In the following, this will be referred to simply as the resonance frequency.

\section{Environmental Test Conditions}

The materials in the device, which are supposed to be most susceptible to radiation damage, are the silicon-dioxide and the piezoelectric AlN. Charge accumulation in these layers could lead to bias of the piezoelectric material. This would
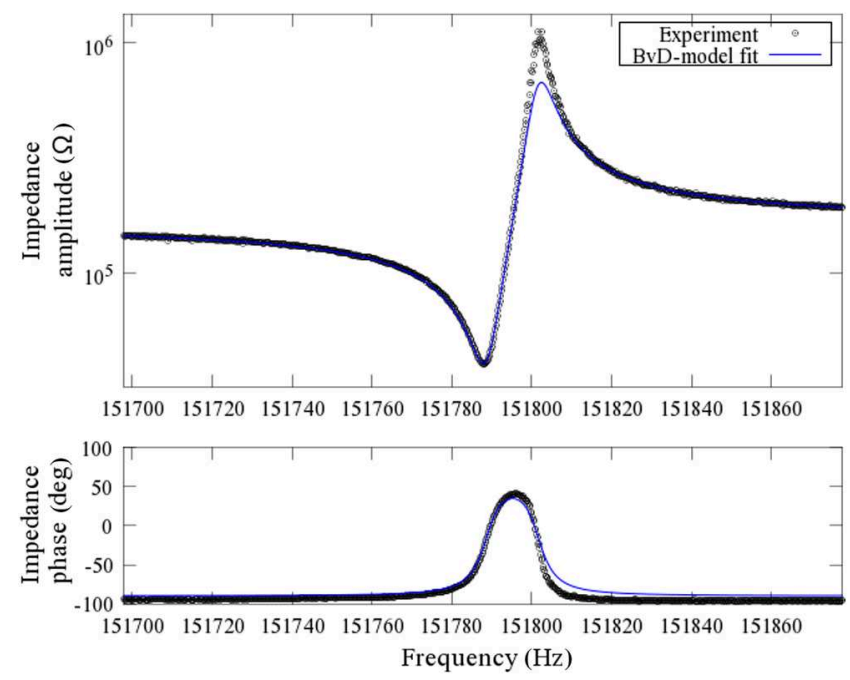

Fig. 5 Impedance absolute value (above) and phase (below) of the resonator near the operation resonance frequency. The black circles are the measurement points and the blue line is a fit of the Butterworth-van-Dyke equivalent circuit. reduce the driving force of the actuator, thus higher driving voltages would be required to drive the resonator. Trapped charges in the piezoelectric layer could affect the resistance and capacitance of the AlN. With regards to the silicon, which makes up for the bulk of the tuning fork tines and dominates their mechanical properties, a displacement-damage induced change in Young's modulus may be possible. Experimental evidence suggests that single-crystal silicon is highly resistant to such an effect, however, the uncertainty in these experiments was significantly larger than in the present study. ${ }^{16,17}$

\section{1 ${ }^{60}$ Co Total lonizing Dose Test}

The irradiation of the resonators with $\gamma$-rays from a ${ }^{60} \mathrm{Co}-$ source was carried out to study the susceptibility of the devices to ionization damage and charge trapping in one of the functional layers. The radiation campaign was carried out at the ${ }^{60} \mathrm{Co}$-irradiation facility of the European Space Agency (ESA) in Noordwijk and was conducted following a radiation test plan based on the European Space Components Coordination (ECSS) radiation test procedure. ${ }^{20} \mathrm{~A}$ total number of 28 devices were irradiated and three devices were used as a nonirradiated control group. The total ionizing doses to which the devices were exposed ranged from $3 \mathrm{krad}(\mathrm{Si})$ to $170 \mathrm{krad}(\mathrm{Si})$. Four samples were irradiated at each irradiation level. Two devices in each group were floating (nondefined potential) and two were biased at $50 \mathrm{mV}$ (defined potential), which corresponded to the AC voltage applied in operation. The devices were electrically characterized directly after the irradiation. The electrical characterization was subsequently repeated after 1 week and after 4 weeks. The devices were annealed at room temperature between the measurements.

\subsection{Proton Irradiation}

Proton irradiation simultaneously inflicts ionizing and nonionizing damages. For radiation tests with protons, it is essential to ensure that the protons fully penetrate the device package and reach the radiation-sensitive parts. The penetration depth increases with the ion energy. On the other hand, the stopping power, which (along with the flux) determines the duration of the irradiation, is inversely related to the ion energy. Therefore, a compromise needs to be found with regards to the penetration depth, the relevant energy range, and the stopping power.

Using SRIM-2008, ${ }^{35}$ the proton irradiation of the packaged devices was simulated. In these simulations, the sample structure consisted of a simplified material stack of $0.5 \mathrm{~mm}$ Pyrex, $20 \mu \mathrm{m}$ air, and $0.48 \mathrm{~mm}$ silicon. The resonators were 
located at the surface of the silicon layer, right below the air gap. A proton energy of $50 \mathrm{MeV}$ resulted in full penetration of the protons into the device and over $99.9 \%$ of ions fully crossed the simulated layers. In the simulations, $10^{6}$ ion impact events were calculated. The effect of the cap was to reduce the energy of the protons on their trajectory toward the silicon microresonator due to the energy losses. As the stopping power increases with decreasing ion energy, the energy loss in the silicon was slightly higher in the shielded devices than it would be in directly irradiated silicon (Fig. 6). However, the difference of only $2 \%$ was within the uncertainty of the beam flux and energy; therefore, the effect of shielding by the cap was negligible.

In total, 19 resonator devices were used for the irradiation campaign. They were repartitioned into six sample groups which were irradiated at different doses (Table 2). One sample group was used as an unirradiated reference. The MEMS components were floating during the irradiation and the postirradiation annealing. In each sample group (except group 2), one device was biased at $200 \mathrm{mV}$ during the irradiation.

The proton irradiations were carried out at the proton irradiation facility of the Paul-Scherrer Institute (Villigen, $\mathrm{CH}$ ). The proton flux was approximately $1 \times 10^{8} \mathrm{p} \mathrm{cm}^{-2} \mathrm{~s}^{-1}$ and the proton energy was $50 \mathrm{MeV}$. At these conditions, the stopping power (i.e., the energy deposited per unit path length) was $0.23 \mathrm{eV} / \AA$ near the irradiated surface (Fig. 6). The

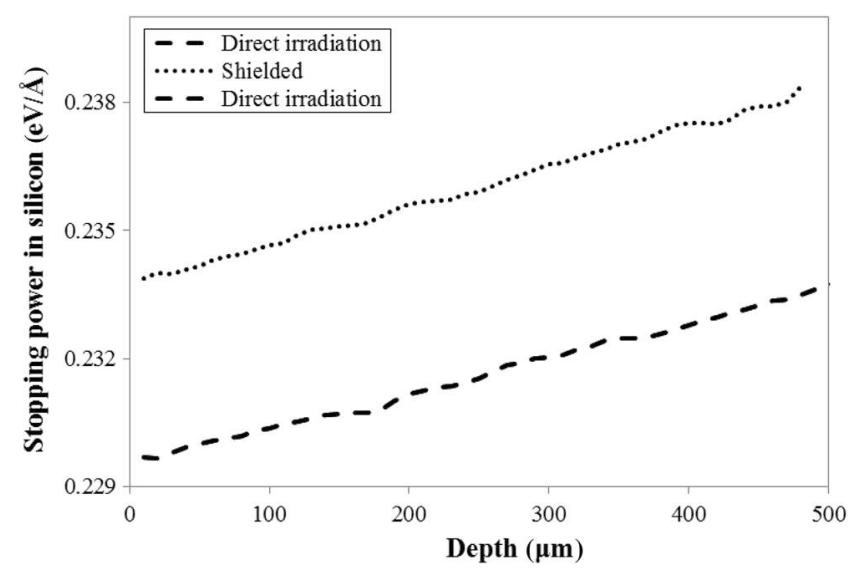

Fig. 6 Comparison of the stopping power (eV/A) of directly irradiated silicon (dotted line) in comparison with the silicon microresonator under the Pyrex cap (dashed line). corresponding dose rate of $16 \mathrm{rad} / \mathrm{s}$ resulted in an irradiation time of $62 \mathrm{~s}$ for $1 \mathrm{krad}$.

The electrical characterization of the devices was made directly after the irradiation and repeated 3 days and 2.5 weeks thereafter. Between the measurements, the devices were annealed at $20 \pm 3^{\circ} \mathrm{C}$.

\subsection{Mechanical Tests}

The aim of the mechanical vibration and shock tests was to evaluate the stability of the devices under the mechanical loads which may be encountered during assembly, transport, and operation. For space applications, the most significant events occur during take-off, stage-separation, and landing. The test procedures carried out were based on the ECSS standard ECSS-E-10-03A $\mathrm{A}^{36}$ and the Military Standard $883 \mathrm{H} .{ }^{37}$

The effect of mechanical shock on the functioning of the resonators was tested using a L.A.B SD-10 free fall shock tester. The specifications of the test procedure are shown in Table 3. For both directions of three orthogonal axes, the samples were subjected to three consecutive shocks so that the total number of shocks at every test level was 18 . After each shock level, the samples were optically inspected and electrical impedance measurements were carried out.

After finishing the mechanical shock tests, the devices were exposed to mechanical vibrations which were based on the ECSS test standard. ${ }^{36}$ The vibration tests were conducted on a TIRAvib TV 50350 instrument. For each of three orthogonal axes, the devices were tested with sinusoidal vibrations followed by random vibrations. Sinusoidal vibrations were applied in the frequency range between 5 and $100 \mathrm{~Hz}$. The test frequency was swept from 5 to

Table 3 Parameters of the mechanical shock tests.

\begin{tabular}{lcc} 
Test level & Peak acceleration $(\mathrm{g})$ & Pulse duration $(\mathrm{ms})$ \\
\hline 1 & 500 & 1 \\
2 & 1500 & 0.5 \\
3 & 2000 & 0.3 \\
4 & 3500 & 0.3 \\
\hline \hline
\end{tabular}

Table 2 Sampling of piezoelectric resonators in the proton irradiation.

\begin{tabular}{|c|c|c|c|c|c|c|}
\hline & Proton energy $(\mathrm{MeV})$ & Flux $\left(\mathrm{cm}^{-2} \mathrm{~s}^{-1}\right)$ & Fluence $\left(\mathrm{cm}^{-2}\right)$ & TID (krad) & \# resonators tested & Comment \\
\hline Group 1 & 50 & $1 \times 10^{8}$ & $6.3 \times 10^{10}$ & 10 & 4 & 1 biased $(200 \mathrm{mV})$ \\
\hline Group 2 & 50 & $1 \times 10^{8}$ & $1.3 \times 10^{11}$ & 20 & 2 & \\
\hline Group 3 & 50 & $1 \times 10^{8}$ & $2.5 \times 10^{11}$ & 40 & 3 & 1 biased $(200 \mathrm{mV})$ \\
\hline Group 4 & 50 & $1 \times 10^{8}$ & $5.0 \times 10^{11}$ & 80 & 4 & 1 biased $(200 \mathrm{mV})$ \\
\hline Group 5 & 50 & $1 \times 10^{8}$ & $9.5 \times 10^{11}$ & 150 & 3 & 1 biased $(200 \mathrm{mV})$ \\
\hline Group 6 & - & - & - & - & 3 & Control \\
\hline
\end{tabular}


Table 4 Parameters of the sinusoidal vibration tests. The test was made without notching. The parameters were adopted from the ECSS-E-10-03A standard. ${ }^{36}$

\begin{tabular}{lc} 
Frequency $(\mathrm{Hz})$ & Test level \\
\hline 5 to 21 & $22 \mathrm{~mm}$ (peak-to-peak) \\
21 to 60 & $20 \mathrm{~g}(0$ to peak) \\
60 to 100 & $6 \mathrm{~g}(0$ to peak) \\
\hline \hline
\end{tabular}

$100 \mathrm{~Hz}$ and back at 2 octaves/min and the acceleration parameters used are given in Table 4. In the frequency range between 20 and $2000 \mathrm{~Hz}$, random vibration was applied. A root mean square acceleration of $37 \mathrm{~g}$ was imposed during $2.5 \mathrm{~min}$ for each axis.

\section{Results}

No change of the optical appearance was observed after the irradiation tests. The resonance characteristics of the devices after ${ }^{60} \mathrm{Co}$ irradiation and after proton irradiation are shown in Figs. 7 and 8, respectively. In both figures, the left graph displays the relative change of the resonance frequency while the right graph shows the absolute change of the quality factor relative to the state before irradiation. Each data point in the graphs corresponds to one resonator measured at one time after the irradiation. The areas shaded in gray limit the values of $\Delta f_{\text {res }} / f$ and $\Delta Q$ which lie within one standard deviation of the repeatability uncertainty of the measurement technique. (This mode of presenting the data was chosen in order to improve the readability of the graphs. The width of the shaded area corresponds to the error bars on each of the measurements; hence, the data points within this area did not differ from the initial value by more than the repeatability uncertainty).

The irradiation did not induce a significant change in any of the measured parameters neither for the proton irradiation nor for the $\gamma$-rays. No difference was found between the floating and the biased devices. Moreover, also after 1 week and after 4 weeks no deviations in the resonances were found.

Although the resonance frequencies and quality factors measured after irradiation were mostly within one standard
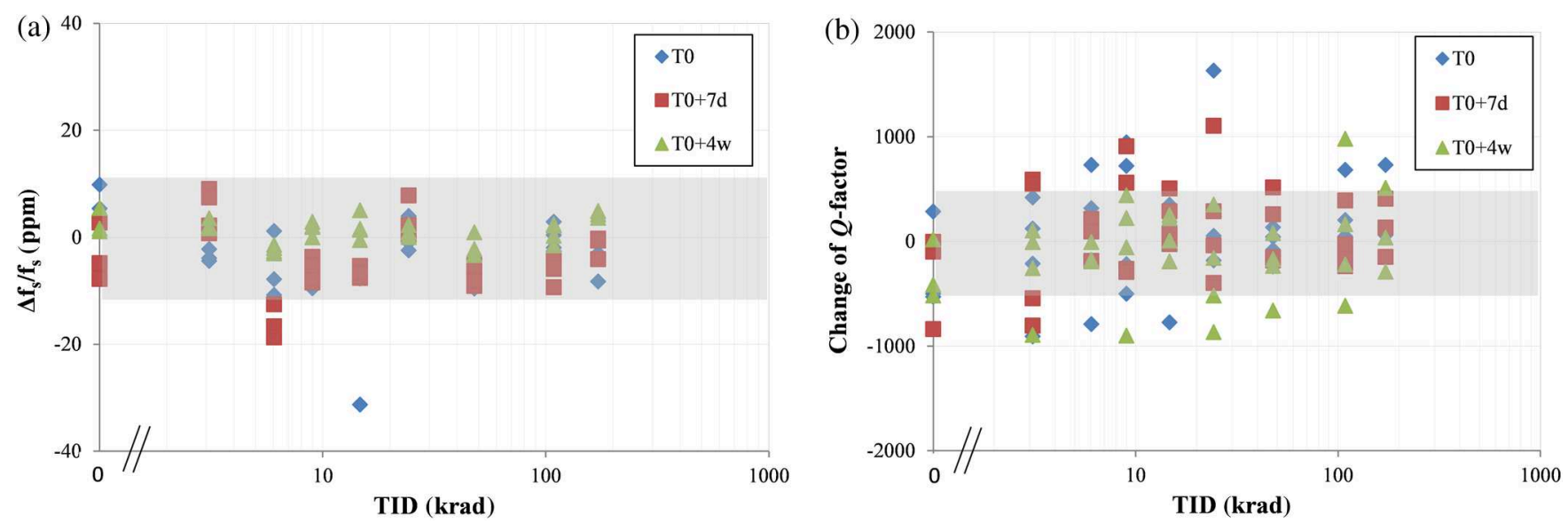

Fig. 7 Resonance characteristics of the tuning fork resonators after irradiation with ${ }^{60} \mathrm{Co} \gamma$-rays. The $x$ axis denotes the absorbed dose. (a) Relative change in the resonance frequency. (b) Absolute change of the quality factor, relative to the preirradiation value, which was about 20,000. ( $\diamond$ Directly after the irradiation, $\square 7$ days after the irradiation, and $\triangle 4$ weeks after the irradiation).
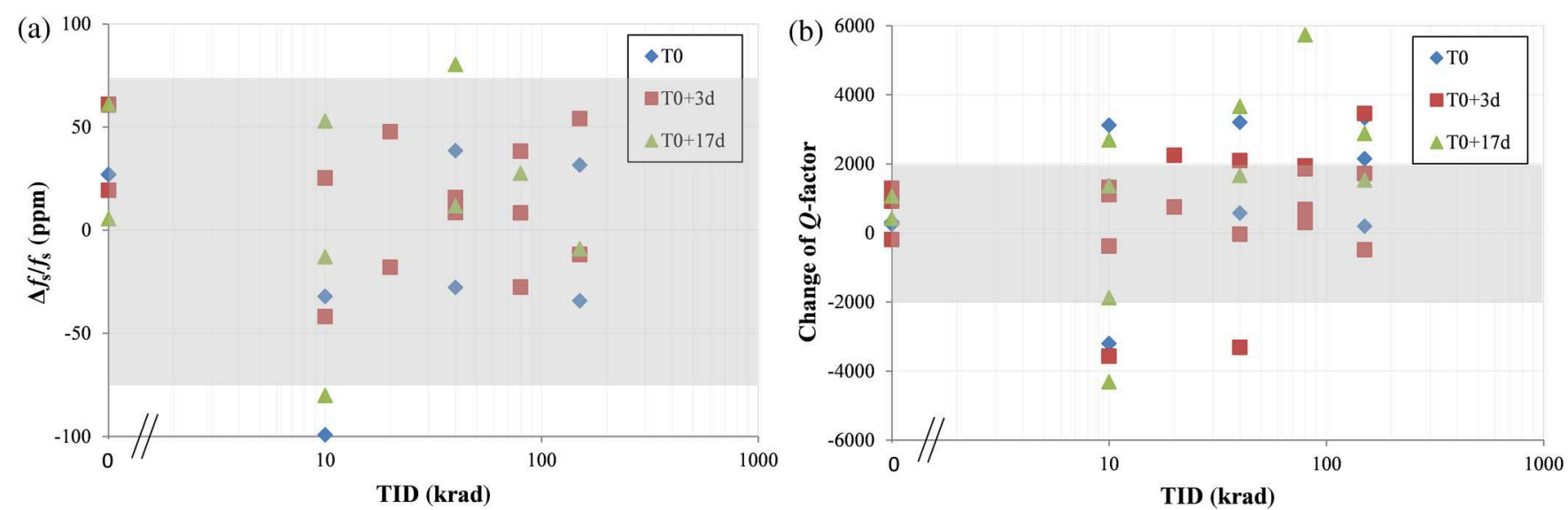

Fig. 8 Relative change of the antiresonance frequency (a) and absolute change of the quality factor (b) during the proton irradiation campaign ( $\diamond$ Directly after the irradiation, $\square 3$ days after the irradiation, and $\triangle 17$ days after the irradiation). 

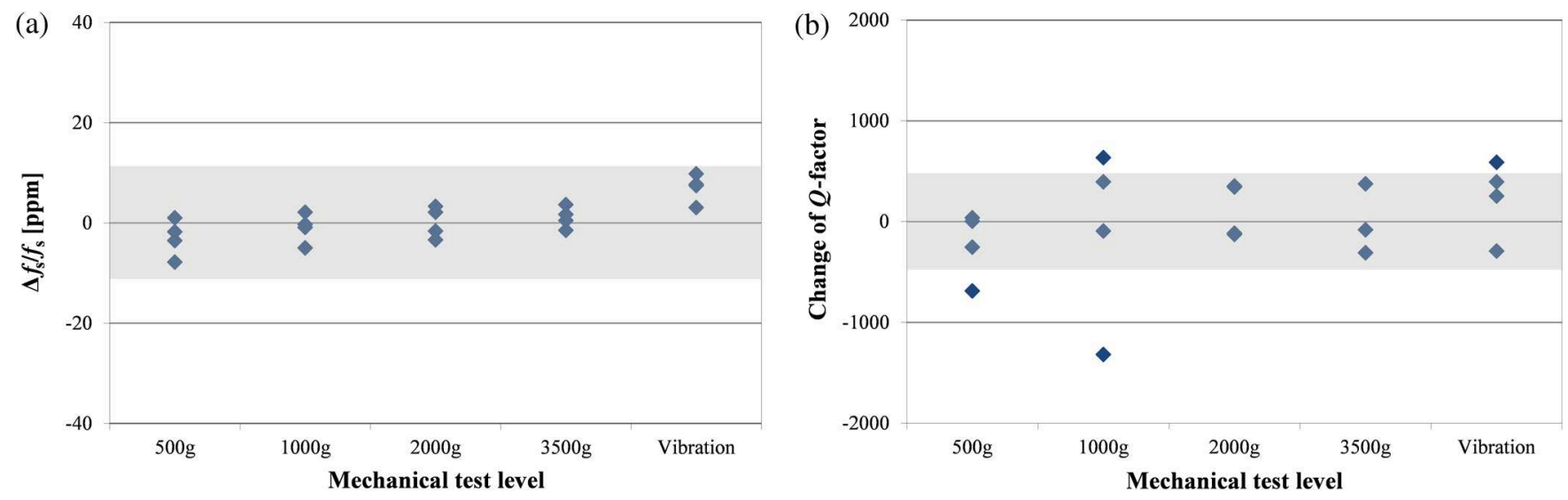

Fig. 9 Resonance characteristics of the tuning fork resonators after mechanical shock and vibration tests. (a) Relative change in the resonance frequency. (b) Absolute change of the quality factor.

deviation of the experimental repeatability uncertainty, the irradiated devices showed a higher scattering of the measurement points than the control specimen, especially the quality factors in the proton irradiation tests (Fig. 8). Further experiments, preferably at higher doses, would be required to assess the repeatability of this observation and clarify the fundamental processes by which radiation damage is induced and accumulated in the system. Nevertheless, these results indicate that the tuning fork resonators are highly resistant to ionizing damage and may perform well under radiation conditions relevant for space applications.

\subsection{Mechanical Tests of Tuning Fork Resonators}

Figure 9 shows the resonance frequency and quality factor of the tested devices after each test level. Again, the left graph shows the relative change of the resonance frequency and the right graph shows the absolute change of the quality factor, relative to the state before irradiation $(\sim 20,000)$. Each data point in the graphs corresponds to one resonator, measured after given mechanical test levels. The areas shaded in gray limit the values of $\Delta f_{\text {res }} / f$ and $\Delta Q$ which lie within one standard deviation of the repeatability uncertainty of the measurement.

The results obtained indicate that the mechanical loads have not caused a significant degradation of the devices and that the specimens have remained unaffected by the tests.

\section{Discussion}

The piezoelectrically activated tuning fork resonators present a very good immunity to irradiation both for ionizing radiation and displacement damage effects. The resonance frequencies did not significantly deviate from the preirradiation values within the experimental uncertainty, which were \pm 11 and \pm 75 ppm for the $\gamma$-ray tests and the proton tests, respectively. This radiation tolerance is explained by the fact that the MEMS device is not dependent on electrostatic actuation, where charge trapping by dielectrics can induce serious degradation of the device performance. ${ }^{1}$ In the tuning fork devices tested here, a charge trapping could lead to screening of the actuation voltage. However, only small energies are required to actuate the tuning fork in its resonance, and the doses tested did not induce measureable deteriorations of the actuation mechanism. The results also confirmed that the ionizing damage and displacement damage have only little effect on the Young's modulus of the materials, most notably on the silicon which accounts for the bulk of the tuning fork tines and dominates the mechanical properties of the resonators.

The quality factors of the resonators did not significantly shift either, however, the repeatability uncertainty was not optimal; the measurement uncertainty was about $3 \%$ of the absolute $Q$-factor value for the $\gamma$-ray tests and $12 \%$ for the proton tests. This explains the higher variation of the $Q$-factor deviations in the proton irradiation. It would be of great interest to further study the influence of radiation on the damping in MEMS materials and the underlying mechanisms. Resonators with high quality factors, and hence minimized intrinsic damping, would be especially suited for this purpose. $^{38}$

The mechanical tests of the resonators have shown that the devices are resistant to high levels of mechanical shocks of up to $3500 \mathrm{~g}$ and vibrations of up to $37 \mathrm{~g}_{\mathrm{rms}}$ and no variations of the resonance frequency or the quality factor were observed within the measurement uncertainty $( \pm 11 \mathrm{ppm}$ and 450 for the resonance frequency and the quality factor, respectively).

\section{Conclusions}

In this contribution, the effect of space-relevant radiation and mechanical loads on silicon tuning fork resonators driven by piezoelectric AIN has been investigated. It was shown that the devices were highly resistant to radiation-induced degradations of doses of up to $170 \mathrm{krad}(\mathrm{Si})$ up to which no degradation was observed within the experimental uncertainty. The devices were immune to mechanical shocks of up to $3500 \mathrm{~g}$ and vibrations of $37 \mathrm{~g}_{\mathrm{rms}}$.

Accelerated aging tests at elevated temperatures were not part of this work and the matter of temperature-related degradation of the devices remains unanswered. However, silicon resonators with very low aging and high resistance to temperature variations have been demonstrated. ${ }^{39}$ The main difference in the qualification processes between systems for space applications and Earth-bound applications are related to radiation, which has been studied here. This work, therefore, supports the efforts taken toward design and fabrication of highly reliable MEMS devices and demonstrates that silicon tuning fork resonators may be well suited for 
space applications from the perspective of radiation tolerance and resistance to mechanical loads.

\section{Acknowledgments}

This work was supported in part by the Networking/ Partnering Initiative of the ESA under Contract No. 21872, by the ESA projects WALES and the Swiss National Science Foundation. We thank Laurent Marchand and Nicolas Saillen from the Components Technology and Space Materials Division of the ESA for the continued project support and collaboration. We kindly acknowledge the support of the members of ESA TEC-QEC: Michele Muschitiello and Alessandra Costantino for the support during the ${ }^{60}$ Co-irradiation tests, Christian Poivey for helpful discussions on the radiation test planning and Michele Brondi, Nat Carthew, and Anthony Denham for the support during the mechanical tests and the electrical characterization. We thank Hajdas Wojtek and Radoslaw Marcinkowski from the Paul-Scherrer Institute for their support during the proton irradiation campaign.

\section{References}

1. O. Colpen and H. E. Gruen, "Tuning fork precision oscillators," in Proc. 13th Annu. Symp. on Frequency Control, IEEE (1959).

2. C.-E. Guillaume, "Nobel lecture-Invar and Elinvar," Nobel Media AB (2013), http://www.nobelprize.org/nobel_prizes/physics/laureates/1920/ guillaume-lecture.html (14 November 2013).

3. J. T. M. van Beek and R. Puers, "A review of MEMS oscillators for frequency reference and timing applications," J. Micromech. Microeng. 22(1), 013001 (2012).

4. Maxim Integrated, "DS3231M \pm 5 ppm, I2C Real-Time Clock," http:// www.maximintegrated.com/datasheet/index.mvp/id/6861 (4 March 2014).

5. SiTime Corp., "SiT1542," http://www.sitime.com/products/32-khzoscillators/sit1542\#magictabs_2nwuQ_1 (4 March 2014).

6. Fortiming Corp., "32.768 kHz watch crystals in cylindrical package WXC15, WXC26, WXC38," http://www.4timing.com/specification/ wxcp.pdf (4 March 2014).

7. Silicon Labs, "Si501/2/3 $32 \mathrm{kHz}-100 \mathrm{MHz}$ CMEMS Oscillator," http://www.silabs.com/Support\%20Documents/TechnicalDocs/Si5012-3.pdf (4 March 2014)

8. Seiko Epson Corp., "RX-4574LC," http://www5.epsondevice.com/en/ quartz/product/rtc/serial3w/rx4574lc.html (4 March 2014).

9. Micro Crystal AG, "CM8V-T1A," http://www.microcrystal.com/ images/_PDF/3_Crystal_Ceramic-Package/cm8v-tla.pdf (4 March 2014).

10. D. Ruffieux et al., "A versatile timing microsystem based on wafer-level packaged XTAL/BAW resonators with sub $\mu \mathrm{W}$ RTC mode and programmable HF clocks," IEEE J. Solid State Circuits 49(1), 212-222 (2014).

11. D. Ruffieux et al., "Silicon resonator based $3.2 \mu \mathrm{W}$ real time clock with \pm 10 ppm frequency accuracy," IEEE J. Solid State Circuits 45(1), 224234 (2010).

12. H. R. Shea, "Radiation sensitivity of microelectromechanical system devices," J. Micro Nanolith. MEMS MOEMS 8(3), 031303 (2009).

13. F. L. Walls and J. R. Vig, "Fundamental limits on the frequency stabilities of crystal oscillators," IEEE Trans. Ultrason. Ferroelect. Freq. Contr. 42(4), 576-589 (1995).

14. P. Gkotsis et al., "Effects of fast neutrons on the electromechanical properties of materials used in microsystems," J. Microelectromech. Syst. 21(6), 1471-1483 (2012).

15. L. Wang, J. Tang, and Q.-A. Huang, "Gamma and electron beam irradiation effects on the resistance of micromachined polycrystalline silicon beams," Sens. Actuators A 177, 99-104 (2012).

16. J. Gomes and H. R. Shea, "Displacement damage effects in silicon MEMS at high proton doses," Proc. SPIE 7928, $79280 \mathrm{G}$ (2011).

17. T. Bandi et al., "Proton-radiation tolerance of silicon and SU-8 as structural materials for high-reliability microsystems," J. MEMS 22(6), 1395-1402 (2013).

18. E. G. Stassinopoulos and J. P. Raymond, "The space radiation environment for electronics," Proc. IEEE 76(11), 1423-1442 (1988).

19. R. A. Mewaldt, "Galactic cosmic ray composition and energy spectra," Adv. Space Res. 14(10), 10737-10747 (1994).

20. ESCC Basic Specification No. 22900, "Total dose steady-state irradiation test method," Issue 3 (2007), https://escies.org (16 February 2012).

21. A. L. Hartzell, M. G. da Silva, and H. R. Shea, MEMS Reliability (MEMS Reference Shelf Series), sec. 4.4.1, Springer US, Boston Mass. (2011).
22. H. Spieler, "Introduction to radiation-resistant semiconductor devices and circuits," AIP Conf. Proc. 390, 23-49, IOP Institute of Physics Publishing LTD (1997).

23. T. R. Oldham, "Analysis of damage in MOS devices for several radiation environments," IEEE Trans. Nucl. Sci. 31(6), 1236-1241 (1984).

24. J. R. Schwank et al., Radiation Hardness Assurance Testing of Microelectronic Devices and Integrated Circuits: Radiation Environments, Physical Mechanisms, and Foundations for Hardness Assurance," SANDIA National Laboratories document SAND-2008-6851P, http:// www.sandia.gov/mstc/services/documents/Sandia_RHA_Foundations_ FINAL.pdf (27 February 2011).

25. M. J. Berger et al., "ESTAR, PSTAR, and ASTAR: Computer Programs for Calculating Stopping-Power and Range Tables for Electrons, Protons, and Helium Ions, Version 1.2.3 (2005)," National Institute of Standards and Technology, Gaithersburg, MD, http://physics.nist .gov/Star (27 February 2011).

26. G. P. Summers et al., "Damage correlation in semiconductors exposed to gamma, electron and proton irradiation," IEEE Trans. Nucl. Sci. 40(6), 1372-1379 (1993).

27. A. Akkerman et al., "Updated NIEL calculations for estimating the damage induced by particles and $\gamma$-rays in Si and GaAs," Radiat. Phys. Chem. 62(4), 301-310 (2001).

28. ASTM International, "Standard guide for use of an $\mathrm{x}$-ray tester $(10 \mathrm{keV}$ photons) in ionizing radiation effects testing of semiconductor devices and microcircuits," http://enterprise.astm.org/filtrexx40.cgi?+REDLINE_ PAGES/F1467.htm (20 June 2012).

29. J. R. Schwank et al., "Optimum laboratory radiation source for hardness assurance testing," IEEE Trans. Nucl. Sci. 48(6), 2152-2157 (2001).

30. P. Paillet et al., "Total dose hardness assurance testing using laboratory radiation sources," IEEE Trans. Nucl. Sci. 50(6), 2310-2315 (2003).

31. C. Muller et al., "Experimental evidence of thermoelastic damping in silicon tuning fork," Proc. Chem. 1(1), 1395-1398 (2009).

32. J. Baborowski et al., "Piezoelectrically activated silicon resonators," IEEE Int. Frequency Control Symp., 2007 Joint with the 21st European Frequency and Time Forum, pp. 1210-1213, IEEE (2007)

33. J. Baborowski et al., "Wafer level packaging technology for silicon resonators," Proc. Chem. 1(1), 1535-1538 (2009).

34. J.-M. Friedt and E. Carry, "Introduction to the quartz tuning fork," Am. J. Phys. 75(5), 415-422 (2007).

35. J. F. Ziegler, J. P. Biersack, and U. Littmark, Stopping and Range of Ions in Solids, Pergamon Press, NY (1985).

36. European Cooperation for Space Standardization (ECSS), "Standard ECSS-E-10-03A space engineering-testing," (2002), http://ecss.nl (26 October 2011).

37. Defense Logistics Agency Standard MIL-STD-883H, "Test method standard microcircuits," http://www.dscc.dla.mil/Downloads/MilSpec/ Docs/MIL-STD-883/std883.pdf (3 May 2012).

38. R. A. Buser and N. F. de Rooij, "Very high Q-factor resonators in monocrystalline silicon," Sens. Actuators 21, 323-327 (1990).

39. M. Lutz et al., "MEMS oscillators for high volume commercial applications," in Proc. Transducers, IEEE, Lyon, France, pp. 49-52 (2007).

Tobias Bandi received BSc and MSc degrees in nanoscience with a major in physics from the University of Basel in 2007 and 2009, respectively. In 2014, he received his $\mathrm{PhD}$ degree from Ecole Polytechnique Fédérale de Lausanne (EPFL) in Switzerland for his work related to MEMS reliability, which he conducted between CSEM SA in Neuchâtel $(\mathrm{CH})$ and the Microsystems for Space Technologies Laboratory (LMTS) of Professor H.R. Shea at EPFL.

Jacek Baborowski received his PhD degree from Lyon University in 1996. He has been the project manager at CSEM since 2004, with expertise in integration of $\mathrm{Si}$ and functional thin films, piezoelectric MEMS and their packaging, SiP low power MEMS radio, and flexible MEMS. He has authored/co-authored over 40 technical publications book chapters and holds seven patents in the field of thin films and MEMS.

Alex Dommann is head of the Department "Materials Meet Life" of the Empa, the Swiss Federal Laboratories for Materials Science and Technology. In 2005, he was appointed CTO of CSEM SA at Neuchâtel. He was a professor of materials research at the Interstate University of Applied Sciences Buchs, where he was scientific head of the Institute for Microsystems from 1997 to 2004 . He received his PhD degree in solid-state physics in 1988 from ETHZ in Switzerland.

Herbert R. Shea received his PhD degree in physics from Harvard University (1997). After a postdoc developing carbon nanotube FETs at IBM's Watson Research Center, he joined Lucent Technologies' Bell Labs where he became the technical manager of the microsystems technology group. In 2004, he joined the Ecole 
Polytechnique Fédérale de Lausanne (EPFL) in Switzerland, where he is an associate professor. Current research topics include miniaturized elastomer-based actuators and sensors, and MEMS for small spacecraft.

Francis Cardot is currently a project manager in the Microsystems Technology Division at CSEM. He received a doctorate from the University of Grenoble, France, in electrochemistry in 1989. He has considerable experience in the design and fabrication of magnetic, mechanical, and electrochemical sensors and actuators for microsystems, having taken ideas from conception all the way through to production in CSEM's new microsystem production facility at Neuchâtel.

Antonia Neels is heading the Center for X-ray Analytics at Empa, Swiss Federal Laboratories for Materials Science and Technology. She received her PhD degree in chemistry from the University of Neuchâtel $(\mathrm{CH}, 1995)$ and the diploma from the Humboldt University Berlin (D, 1991). She has been leading the XRD Laboratory at the IMT-EPFL and CSEM until 2014. Her research interests include new materials with focus on strain and defect mobility in materials and systems. 